\title{
Anti-mycobacterial recall responses differentiate female patients with genital tuberculosis from patients with other gynecological problems
}

\author{
Markos Abebe ${ }^{1}$, Abraham Aseffa ${ }^{1}$, Morten Harboe ${ }^{1,4}$, Zufan Lakew ${ }^{3}$, Lukman Yusuf ${ }^{3}$, Joseph Olobo ${ }^{1,5}$, Mekuria \\ Lakew $^{2}$
} \begin{abstract}
specimens. mycobacterial antigens. tests. $(\mathrm{p}<0.05)$.

\section{Introduction}

Female genital tuberculosis (FGTB) is one type of extra pulmonary tuberculosis affecting the female genital organs. In $90 \%$ of cases the fallopian tubes are the primary foci of infection. From here the bacilli often disseminate to infect the ovaries (10-30\%), endometrium $(50 \%)$, cervix $(<5 \%)$ as well as the vagina and vulva $(<1 \%)(1,2)$. The most affected groups $(80-90 \%)$ are women between 20 and 40 years of age $(1,3)$.
\end{abstract}

Abstract

Background: Female Genital Tuberculosis (FGTB) is one form of extra pulmonary tuberculosis affecting the female reproductive organs, most commonly the fallopian tubes and the endometrium. It affects young women aged between 20 and 40 years of age and is an important cause of infertility. It often occurs as a secondary complication following pulmonary tuberculosis. Diagnosis depends mainly on clinical suspicion in countries where facilities for mycobacterial culture and histopathology are unavailable. Even in places where these facilities exist, diagnosis still remains difficult because of the lower sensitivity and specificity of the methods as well as the invasive procedure of acquiring biopsy

Objective: To explore the immunological profiles of female genital tuberculosis (FGTB) patients in response to

Methods: Twenty-five clinically suspected cases of FGTB and 12 control subjects who came to the Black Lion hospital for unrelated gynecological problems were included in the study. Peripheral blood samples were collected from each subject. Plasma was separated by centrifugation and PBMC were isolated over ficoll-hypaque and stimulated in vitro with mycobacterial antigens to examine their proliferative response as incorporation of tritiated thymidine using a $\beta$-counter. HIV status and total IgG-, IgA- and IgM- antibody levels were determined by ELISA

Results: In vitro recall responses to M. tuberculosis antigens (PPD and BCG sonicate) as well as plasma levels of IgGIgA- and IgM-antibodies to MPT59 showed statistically significant differences between the patients and the controls

Conclusion: The results show that PBMC of FGTB patients recognize $M$. tuberculosis antigens more strongly than PBMC of patients with other gynecological problems. [Ethiop.J.Health Dev. 2005;19(3):219-224]

Prior to the onset of the HIV epidemic, extra pulmonary TB used to occur in relatively a few (about $15 \%$ ) FGTB cases (4). With the onset of the HIV epidemic, however, the occurrence has increased both in absolute and relative numbers with up to $62 \%$ extra pulmonary involvement in patients with advanced HIV status according to one study (5). Thus, HIV has emerged as a most important risk factor in the progression of new or dormant TB infection to clinical stages of the disease (6).
The most frequent clinical symptoms of FGTB are: infertility, lower abdominal distention and pain, menstrual irregularity, fallopian tube abscess, ectopic pregnancy, weight loss, pelvic mass and signs of TB elsewhere in the body (3).

Diagnosis of FGTB is usually made based on clinical suspicions (7), and in cases where the appropriate facilities are available, microbiological (AFB staining and culture), histopathological (8) and radiological methods (HSG) are deemed valuable $(9,10)$. Also in cases where the required highly skilled personnel and specialized equipment are available, molecular methods such as the Polymerase Chain Reaction (PCR) might be employed to detect mycobacterial DNA using specific primers. The technique is rapid and sensitive (11). In lung tissue PCR for M. tuberculosis DNA is found to be frequently positive during latency (12), and thus it might be difficult to differentiate between latent infection and active disease by PCR. All the above methods target the

\footnotetext{
${ }^{1}$ Armauer Hansen Research Institute (AHRI), Addis Ababa, Ethiopia. Tel.: 251-1-211334; Fax: 251-1-211563; P.O. Box 1005, Addis Ababa, E-mail: markosabebe@yahoo.com; ${ }^{2}$ Addis Ababa University, Science Faculty, Department of Biology; ${ }^{3}$ Addis Ababa University, Medical Faculty, Department of Obstetrics and Gynecology; ${ }^{1,4}$ Institute of Immunology,University of Oslo, Rikshospitalet and Rikshospitalet University Hospital, Oslo, Norway; ${ }^{1,5}$ Makerere University, Kampala, Uganda
} 
organism attempting to directly demonstrate its presence in tissue or reveal morphologically characteristic lesions caused by the bacilli.

An alternative approach is to exploit the specific nature of immune responses to invading pathogens by eliciting the recall response upon secondary exposure in vitro. The use of humoral or cellular responses to detect the presence of tuberculous infection or disease is an area under investigation (13). By using PBMC obtained from FGTB suspected patients and controls, this study attempted to investigate immune responses to mycobacterial antigens (lymphocyte proliferation assay to BCG sonicate and Purified Protein Derivative, PPD, and antibody responses to MPT59, a secreted protein of M. tuberculosis) to assess whether these immunological methods are capable of assisting the diagnosis of FGTB.

\section{Methods \\ Patients}

Thirty-seven women, 25 of whom are suspected to have genital TB on clinical grounds (patients), and 12 controls were included in the study. Most patients came to the hospital and TB was suspected on clinical basis due to infertility problem, and laboratory findings. The controls were gynecological patients who underwent surgery due to other gynecological problems (mainly with cancer) having no clinical signs or symptoms suggestive of tuberculosis. Surgically excised tissue was taken from both groups for laboratory examination, including AFB using light microscopy following concentration and staining by $\mathrm{ZN}$ as well as culture and $M$. tuberculosisspecific PCR (14).

The study was approved by the Ethical Review Committees of AHRI/ALERT, the Department of Obstetrics and Gynecology (Medical Faculty of Addis Ababa University), and the National Ethical Review Committee (Ethiopian Science and Technology Commission). Informed consent was obtained from all the women included in the study.

Antigens: MPT59 (Antigen 85B, Rv1886c, is a major secreted protein in $M$. tuberculosis culture filtrates containing reactive B cell epitopes) $(15,16)$ and BCG sonicate were obtained as previously described (15). PPD was obtained from the Statens Serum Institute, Copenhagen, Denmark, and PHA was purchased from Sigma. The antigens were aliquoted and frozen at $-20^{\circ} \mathrm{C}$ before use.

\section{Isolation of PBMC}

Venous blood $(10 \mathrm{ml})$ was drawn in heparinized tubes, centrifuged, and the plasma separated. The pellet was diluted with RPMI-1640 in a one to one ratio and layered over Ficoll-Hypaque (Pharmacia, Uppsala, Sweden). It was then centrifuged for 30 minutes at $1800 \mathrm{rpm}$ and at room temperature. Peripheral Blood Mononuclear Cells
(PBMC) were collected and washed three times with RPMI-1640, each time centrifuged at $1500 \mathrm{rpm}$ for five minutes at $4^{0} \mathrm{C}$. PBMC were resuspended in two $\mathrm{ml}$ of $5 \%$ Normal Human Serum (NHS) and stored in liquid nitrogen. Plasma was stored frozen at $-20^{\circ} \mathrm{C}$ until it was used.

\section{HIV antibody testing by ELISA}

The study subjects were tested for HIV antibodies using a microelisa system kit (Vironostika, HIV Uni-Form 11 Plus, Organon Teknika GmbH, Eppelheim, The Netherlands in accordance with the manufacturer's instructions.

\section{Lymphocyte Stimulation Test (LST)}

PBMC were cultured in 96 well tissue culture plates (Flow laboratory, Irvine KA1 28NB, Scotland) at a concentration of $10^{5}$ cells/well in the presence of PHA $(1 \mu \mathrm{g} / w e l l)$ or PPD $(10 \mu \mathrm{g} /$ well (Statens Serum Institute, Copenhagen, Denmark) or BCG sonicate in 5\% NHS supplemented with 5\% glutamine and 5\% PenicillinStreptomycin in RPMI as culture medium and incubated at $37^{\circ} \mathrm{C}$ in $5 \% \mathrm{CO}_{2}$. Then, the cells were pulsed with tritiated thymidine (Amersham, Little Chalfont, UK) $(1 \mu \mathrm{Ci} /$ well) at day three (PHA) and day six (BCG and PPD). After $18 \mathrm{hrs}$, the cells were harvested on to a filter mat (cat. No. 11731). Finally, proliferation was measured on a $\beta$-liquid scintillation counter (LKB Wallac 1216 Rackbeta II, Uppsala, Sweden) and the stimulation index (SI) was calculated by dividing the CPM (counts per minute) of antigen stimulated cells with the CPM of non stimulated controls.

\section{ELISA (Enzyme Linked Immuno Sorbent Assay)}

Flat-bottomed 96 well plates (Immulon-2 Dynatech Laboratories, Chantilly, VA, USA) were coated with 0.5 $\mu \mathrm{g} /$ well of purified antigen, MPT59 in phosphate buffered saline (PBS) (pH 7.2) and incubated overnight at $4^{0} \mathrm{C}$. The plates were then washed four times with $0.1 \%$ Tween in PBS (PBS-T) and blocked with $100 \mu$ of blocking buffer (5mg bovine serum albumin $/ \mathrm{ml}$ in PBS) and incubated for $2 \mathrm{hrs}$ at room temperature (RT). Serum samples were diluted 1:50 for IgG- and IgM- and 1:10 for IgA antibody assay and added at $100 \mu \mathrm{l} /$ well and incubated for $1 \mathrm{hr}$ at RT. After washing four times in $0.1 \%$ PBS-T, $100 \mu$ l of peroxidase conjugated secondary antibody (anti-human IgG/IgA/IgM) (Sigma) $(1 \mathrm{mg} / \mathrm{ml}$ ) diluted 1:10,000 was added and incubated for $1 \mathrm{hr}$ at RT. Finally, $100 \mu \mathrm{l} /$ well of the substrate o-Phenylenediamine Dihydrochloride (OPD) was added and incubated for 30 minutes. The reaction was stopped by adding $50 \mu \mathrm{l}$ stop solution $\left(1 \mathrm{M} \mathrm{H}_{2} \mathrm{SO}_{4}\right)$ to each well. The optical density (OD) was read at $492 \mathrm{~nm}$ using an ELISA reader (Titertek Multiskan Plus, Helsinki, Finland).

\section{Statistical analysis}

Statistical analysis was carried out using the Mann- 
Whitney rank sum test. Fisher's exact test was also used to compare proportions. Differences among groups were considered to be significant when the p-value was found to be less than or equal to 0.05 .

\section{Results}

Among the 37 study subjects, 25 were suspected clinically to have FGTB and out of these 25 suspected cases, 16 were confirmed to be positive for $M$. tuberculosis based on laboratory findings (14). The main clinical symptoms detected in the patient group were infertility. Out of the 17 patients whose infertility status was known, six (35\%) had primary and $11(65 \%)$ had secondary infertility. Other symptoms included lower abdominal pain, irregular menstrual bleeding and pelvic mass, (Table 1). The age range of the patients was between 18-39 years and the median age was 28 .

\section{Immunological assays}

HIV status

To determine the HIV status of the study subjects, HIVELISA test was performed for 34 patients (22 patients and 12 controls). The result showed that 15 out of the 34 (43\%) patients were HIV positive. The proportion of seropositivity among patients was found to be $44 \%$ compared to $25 \%$ among the controls $(\mathrm{p}<0.05)$.

\section{Antibody Assay}

An antibody assay was performed using MPT59 as an antigen on the solid phase in the ELISA test. Fig. 1 shows the plasma level of the three antibody isotypes irrespective of their HIV status.

Table 1: Clinical presentation and laboratory findings in 25 women who visited Tikur Anbessa Hospital for infertility problem and who are suspected to have genital tuberculosis

\begin{tabular}{|c|c|c|c|c|c|}
\hline Patient & Signs and Symptoms & AFB & Culture & Histology & PCR \\
\hline $\mathrm{M}-003$ & $2^{0}$ infertility, bilateral tubal blockage & - & - & + & - \\
\hline M-006 & $\begin{array}{l}2^{0} \text { infertility, right tubal blockage, irregular menses, vaginal } \\
\text { bleeding, adhesion }\end{array}$ & - & - & + & - \\
\hline M-007 & $\begin{array}{l}2^{0} \text { infertility, bilateral tubal blockage, irregular menses, pelvic pain, } \\
\text { vaginal discharge, adhesion }\end{array}$ & - & - & - & - \\
\hline$M=012$ & $1^{0}$ infertility & + & + & + & + \\
\hline M-017 & $1^{0}$ infertility, bilateral tubal blockage & - & - & - & + \\
\hline M-020 & $\begin{array}{l}1^{0} \text { infertility, vaginal discharge, adhesion, lower abdominal mass, } \\
\text { pain }\end{array}$ & - & + & - & + \\
\hline M-024 & $2^{0}$ infertility, bilateral tubal blockage, amenorrhoea & - & + & + & + \\
\hline $\mathrm{M}-028$ & $2^{0}$ infertility, bilateral tubal blockage & - & - & - & + \\
\hline M-032 & $2^{0}$ infertility. bilateral tubal blockage, lower abdominal pain & - & - & - & + \\
\hline M-037 & Adhesion & - & - & - & + \\
\hline M-041 & Pelvic pain & - & - & - & - \\
\hline M-045 & $1^{0}$ infertility, bilateral hydrosalpinx, ammenorrhoea & - & - & - & - \\
\hline M-049 & $2^{0}$ infertility, bilateral tubal blockage, vaginal discharge & - & - & - & + \\
\hline M-053 & $2^{0}$ infertility, bilateral tubal blockage & - & - & + & + \\
\hline M-057 & $1^{0}$ infertility & - & - & - & + \\
\hline M-060 & $1^{0}$ infertility & - & - & - & - \\
\hline M-063 & $2^{0}$ infertility, infertility, abdominal swelling & - & - & - & - \\
\hline M-066 & Right tubal blockage, adhesion & - & - & - & + \\
\hline M-069 & Irregular menses, vaginal bleeding, lower abdominal pain & - & - & + & - \\
\hline M-072 & $2^{0}$ infertility & - & - & - & - \\
\hline M-077 & $1^{0}$ infertility & - & - & - & - \\
\hline M-081 & $1^{0}$ infertility, bilateral tubal blockage & - & - & - & - \\
\hline M-086 & $1^{0}$ infertility, bilateral tubal blockage, vaginal discharge, adhesion & - & - & + & - \\
\hline M-089 & $1^{0}$ infertility, bilateral hydrosalpinx, dysmenorrhoea & - & - & - & + \\
\hline \multirow[t]{2}{*}{ M-093 } & $2^{0}$ infertility, tubo-peritionial factor & - & - & - & - \\
\hline & Total positive & 1 & 3 & 7 & 12 \\
\hline
\end{tabular}


Table 1 continued......

\begin{tabular}{|c|c|c|c|c|}
\hline Patient & Signs and Symptoms & TB status & HIV & CD4/CD8 (ratio) \\
\hline $\mathrm{M}-003$ & $2^{0}$ infertility, bilateral tubal blockage & + & - & ND \\
\hline M-006 & $\begin{array}{l}2^{0} \text { infertility, right tubal blockage, irregular menses, vaginal } \\
\text { bleeding, adhesion }\end{array}$ & + & - & ND \\
\hline M-007 & $\begin{array}{l}2^{0} \text { infertility, bilateral tubal blockage, irregular menses, pelvic pain, } \\
\text { vaginal discharge, adhesion }\end{array}$ & - & - & ND \\
\hline$M=012$ & $1^{0}$ infertility & + & ND & ND \\
\hline M-017 & $1^{0}$ infertility, bilateral tubal blockage & + & + & ND \\
\hline M-020 & $\begin{array}{l}1^{0} \text { infertility, vaginal discharge, adhesion, lower abdominal mass, } \\
\text { pain }\end{array}$ & + & ND & ND \\
\hline M-024 & $2^{0}$ infertility, bilateral tubal blockage, amenorrhoea & + & + & ND \\
\hline M-028 & $2^{0}$ infertility, bilateral tubal blockage & + & - & $27 / 33(0.8)$ \\
\hline M-032 & $2^{0}$ infertility. bilateral tubal blockage, lower abdominal pain & + & + & $37 / 14(2.6)$ \\
\hline M-037 & Adhesion & + & + & $23 / 53(0.4)$ \\
\hline M-041 & Pelvic pain & - & + & $51 / 18(2.8)$ \\
\hline M-045 & $1^{0}$ infertility, bilateral hydrosalpinx, ammenorrhoea & - & - & $36 / 38(0.9)$ \\
\hline M-049 & $2^{0}$ infertility, bilateral tubal blockage, vaginal discharge & + & - & ND \\
\hline M-053 & $2^{0}$ infertility, bilateral tubal blockage & + & - & $42 / 42(1)$ \\
\hline M-057 & $1^{0}$ infertility & + & - & $41 / 33(1.2)$ \\
\hline M-060 & $1^{0}$ infertility & & + & $20 / 51(0.4)$ \\
\hline M-063 & $2^{0}$ infertility, infertility, abdominal swelling & & - & $21 / 30(0.7)$ \\
\hline M-066 & Right tubal blockage, adhesion & + & - & $44 / 30(1.5)$ \\
\hline M-069 & Irregular menses, vaginal bleeding, lower abdominal pain & + & + & $33 / 28(1.2)$ \\
\hline M-072 & $2^{0}$ infertility & - & + & ND \\
\hline M-077 & $1^{0}$ infertility & - & ND & $10 / 20(1)$ \\
\hline M-081 & $1^{0}$ infertility, bilateral tubal blockage & - & + & $34 / 35(1)$ \\
\hline M-086 & $1^{0}$ infertility, bilateral tubal blockage, vaginal discharge, adhesion & + & - & $30 / 39(0.8)$ \\
\hline M-089 & $1^{0}$ infertility, bilateral hydrosalpinx, dysmenorrhoea & + & - & ND \\
\hline M-093 & $2^{0}$ infertility, tubo-peritionial factor & - & + & ND \\
\hline & Total positive & 16 & 10 & \\
\hline
\end{tabular}

$\mathrm{ND}=$ not done; $\mathrm{AFB}=\mathrm{Acid}$ Fast Bacilli

All the clinically suspected FGTB cases were found to be reactive (IgG, IgA, IgM) to this antigen with variably high levels of ODs. The difference in median antibody levels between the two groups was significantly different for all the three immunoglobulins: $\operatorname{IgG}, \operatorname{IgA}$ and $\operatorname{IgM}(\mathrm{p}<0.05)$. The data were analyzed based on HIV status, and showed that there was no statistically significant difference between HIV positive and HIV negative individuals regarding the antibodies of the three isotypes $(\mathrm{p}=0.6)$.

\section{Lymphocyte Stimulation Test (LST)}

PBMC were subjected to LST test using the mitogen phytohaemaglutinin (PHA) as a control for proper cell viability and the mycobacterial antigens BCG sonicate and PPD. Fig. 2 shows the proliferative response of patients and controls to these antigens irrespective of their HIV status.
Although there was a variation in the degree of response to stimulation, all subjects responded well to PHA, mean SI = 39 in patients and SI $=31$ in controls. This difference was not significant $(\mathrm{p}>0.05)$. There was however, a statistically significant difference between the patient and the control groups in response to both BCG $(\mathrm{p}=0.03)$ and PPD ( $=$ $0.025)$.

\section{Discussion}

Although the magnitude of the prevalence of FGTB in Ethiopia is not known, it will remain an important gynecological problem as long as pulmonary TB is rampant. PBMC from FGTB patients react to mycobacterial antigens more strongly than PBMC from the controls (patients with other gynecological problems, mainly cervical cancer). This shows that although FGTB is a localized disease, there are 


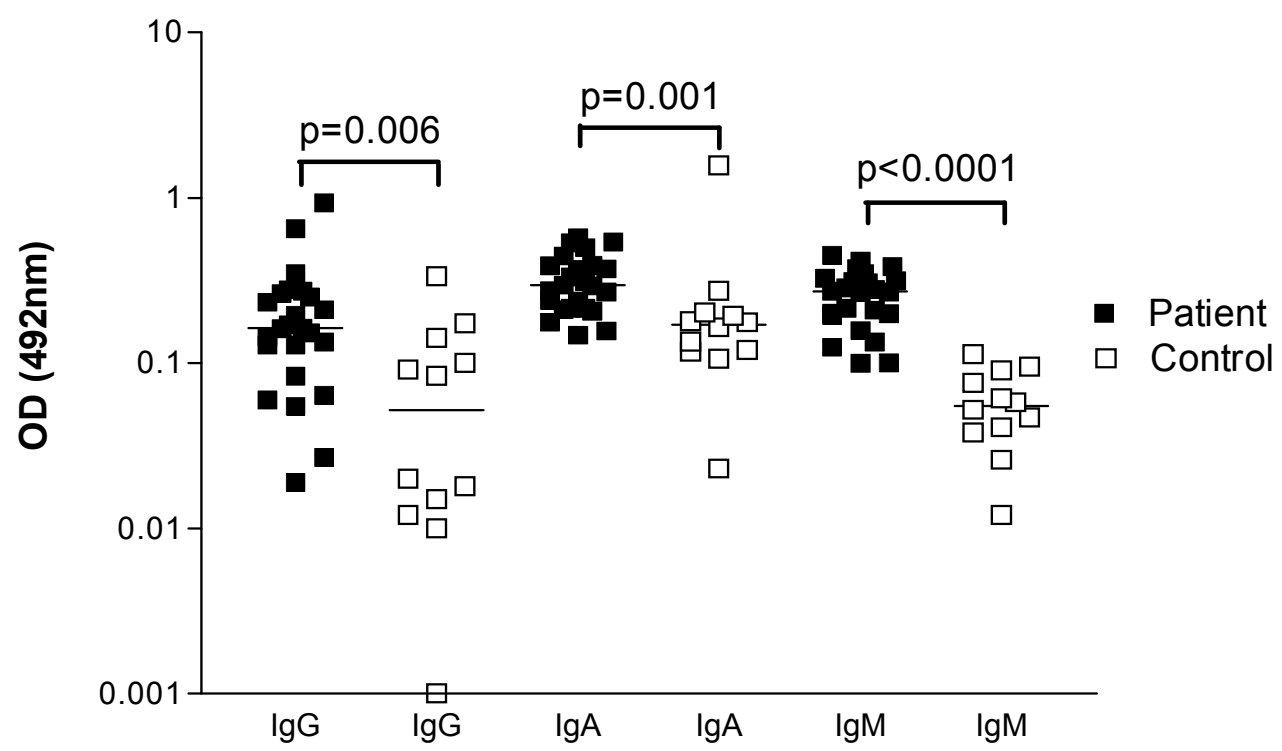

Figure 1: Levels of antibodies to MPT 59 antigen in the plasma of patients suspected to have female genital tuberculosis and the control gynecological patients, Addis Ababa, 2002

OD=Optical Density

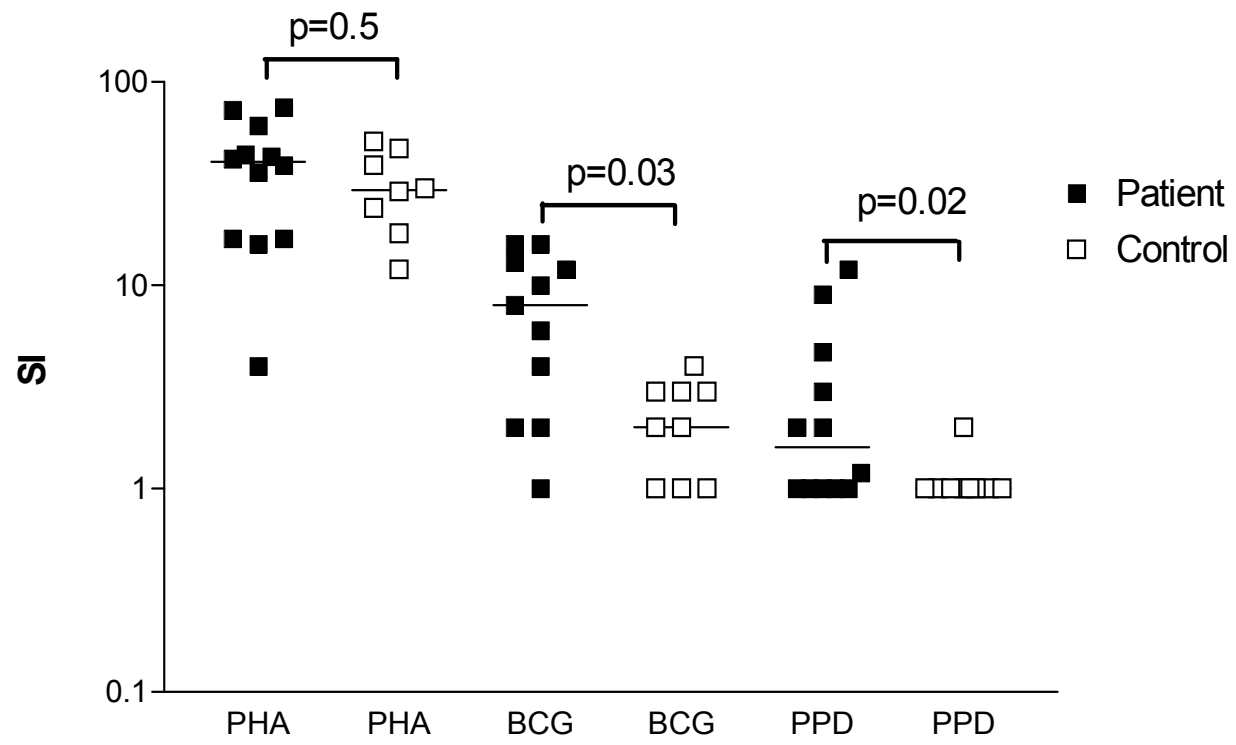

Figure 2: Proliferative response to stimulation with mycobacterial antigens of PBMC from patients suspected to have female genital tuberculosis and control gynecological patients, Addis Ababa, 2002.

SI=stimulation Index PBMC=Peripheral Blood Mononuclear cells

circulating memory $\mathrm{T}$ and $\mathrm{B}$ cells in the peripheral blood, which could be targeted for immune based diagnostic methods.

Plasma from all the 25 patients clinically suspected of suffering from FGTB contained antibodies to the mycobacterial antigen, MPT59. Another study by Parikh et al. conducted in Indian women (17) has produced similar results. This study confirms that all of their 20 clinically diagnosed FGTB patients had IgG-, IgM- or
IgA antibodies to a different type of $M$. tuberculosis antigen denoted as A60. The $100 \%$ reactivity was achieved when the three antibody isotypes were tested in each individual patient since a patient without IgG antibodies could have IgM- or IgA antibodies and vice versa. Antibody positivity does not, however, indicate which organ is affected by tuberculosis.

A similar result was obtained concerning the proliferative response of PBMC from patients to PPD and BCG. The 
SI of patients was significantly higher than that of the control group to both PPD $(\mathrm{p}=0.02)$ and $\mathrm{BCG}(\mathrm{p}=$ $0.03)$.

One limitation in the study is the relatively small sample size employed and the fact that most of the controls were cancer patients. This could not be avoided because it is only from such patients that a biopsy material could be obtained for investigation. It is also possible that the weaker immune response seen in the control group could be due to a possibly immunocompromised status in these individuals. Another confounder could be the expected different levels of progression in the HIV- infected individuals in the two groups. This is probably not the case, as the difference in the CD4:CD8 ratios in the patients and the controls were found to be statistically significant.

FGTB patients are often diagnosed lately because of the lack of early detection methods, difficulties with confirmatory tests, and the need for surgical intervention to obtain the appropriate specimens for diagnosis. Early detection is crucial for the proper management of FGTB. Since diagnosis on clinical grounds alone is unreliable, less invasive techniques than surgical interventions are needed. Tests based on the immune response patterns of the subjects using PBMC would be helpful in this regard. The findings of this study also provide the baseline information that PBMC from FGTB patients recognize mycobacterial antigens more strongly than cells from patients with other gynecological problems. Further studies are required to test whether immune response assays could serve as additional diagnostic tests in situations where the confirmation of localized tuberculosis becomes difficult using more thoroughly screened controls and additional selected M. tuberculosis antigens.

\section{Acknowledgments}

The authors of this study acknowledge the assistance of Dr. Taddesse Kitila in clinical diagnosis and in the provision of biopsy specimens used in the study and Sr. Atsede Tesfamariam for drawing blood samples. NORAD and SIDA are acknowledged for their financial support to the core budget of AHRI, which supported this work.

\section{References}

1. Varma TR. Genital tuberculosis and subsequent fertility. Int J Gynecol Obstet 1991;35:1-11.
2. Arora VK, Johri A, Arora R, Rajaram P. Tuberculosis of the vagina in an HIV seropositive case. Tuber Lung Dis 1994;75:239-240.

3. Crofton J, Horne N, Miller F. Clinical tuberculosis. $1^{\text {st }}$ ed. MACMILLAN Education LTD. London, 1992.

4. Farer LS, Lowell LM, Meador MP. Extrapulmonary tuberculosis in the United states. Am J Epidemiol 1979; 109:205-217.

5. Small PM, Schecter GF, Goodman PC, Sande MA, Chaisson RE, Hopewell PC. Treatment of tuberculosis in patients with advanced human immunodeficiency virus infection. N Eng J Med 1991; 324:289-294.

6. Sehgal PN. TB and AIDS. Health millions 1995; 21:34-35.

7. Simon HB, Weinstein AJ, Pasternak MS, Swarz MN, Kunz LJ. Genitourinary tuberculosis: Clinical features in a general hospital population. Am J Med 1977; 63:410-420.

8. Winfred JA. Female genital tuberculosis. J Obstet Gynecol 1977; 63:418-428.

9. Schaefer G. Female genital tuberculosis. Clin Obstet Gynecol 1976; 19:223-239.

10. Rom W, Garay S. Tuberculosis. $1^{\text {st }}$ ed. New York: Little Brown and Company (inc.) 1996.

11. Bloom BR, Murray CJ. Tuberculosis: Commentary on a re-emergent killer. Science 1992;257:1055-1064.

12. Hernández-Pando R, Aguilar D, Orozco H, Jeyanathan M, Rook G A W, Mengistu G, Harboe M, et. al. Persistence of DNA from Mycobacterium

tuberculosis in superficially normal lung tissue during latent infection. The Lancet 2000; 356:2133-2138.

13. Demissie A, Abebe M, Aseffa A, Rook G, Fletcher H, Zumla A, et al. Healthy individuals that control a latent infection with Mycobacterium tuberculosis express high levels of Th1 cytokines and the IL-4 antagonist IL-482. J Immunol 2004;172:6938-6943.

14. Abebe M, Lakew M, Kidane D, Lakew Z, Kiros K, Harboe M. Female genital tuberculosis in Ethiopia. Int J Gynecol Obstet 2004;84:241-246.

15. Nagai S, Wiker HG, Harboe M, Kinomoto M. Isolation and partial characterization of major protein antigens in the culture fluid of Myco-bacterium tuberculosis. Infect Immun 1991;59: 372-382.

16. Wiker HG, Harboe M. The antigen 85 complex: A major secretion product of Mycobacterium tuberculosis. Micrbiol Rev 1992;56:648-661.

17. Parikh FR, Nadkarini SG, Kamat SA, Naik N, Soonawala SB, Parikh RM. Genital tuberculosis - a major pelvic factor causing infertility in Indian women. Fertil Steril 1997;67:497-500. 
